\title{
Erratum to: An enhanced beam-theory model of the mixed-mode bending (MMB) test-Part I: Literature review and mechanical model
}

\author{
Stefano Bennati · Paolo Fisicaro • Paolo S. Valvo
}

Published online: 17 January 2013

(C) Springer Science+Business Media Dordrecht 2013

\section{Erratum to: Meccanica}

DOI 10.1007/s11012-012-9686-3

Due to an unfortunate turn of events this article was published with an erroneous version of Eqs. (31) and (47). Please find on this page the correct version of Eqs. (31) and (47) that should be regarded by the reader as the final version.
$\left.N_{1}^{(\mathrm{s})}\right|_{s=s_{A}}=0$

$\left.Q_{1}^{(\mathrm{s})}\right|_{s=s_{A}}=P_{I}$,

$\left.M_{1}^{(\mathrm{s})}\right|_{s=s_{A}}=0$,

$\left.N_{1}^{(\mathrm{s})}\right|_{s=0^{-}}=\left.N_{1}^{(s)}\right|_{s=0^{+}}$,

$\left.Q_{1}^{(\mathrm{s})}\right|_{s=0^{-}}=\left.Q_{1}^{(\mathrm{s})}\right|_{s=0^{+}}$,

$\left.M_{1}^{(\mathrm{s})}\right|_{s=0^{-}}=\left.M_{1}^{(\mathrm{s})}\right|_{s=0^{+}}$,

$\left.Q_{1}^{(\mathrm{s})}\right|_{s=s_{C}}=0$,

$\left.M_{1}^{(\mathrm{s})}\right|_{s=s_{C}}=0$.

$$
\begin{aligned}
\tau= & -\frac{2}{B h} \lambda_{5}\left(B_{6} \sinh \lambda_{5} s+B_{7} \cosh \lambda_{5} s\right) \\
& +\frac{A_{1} h}{2 B D_{1}} B_{8} .
\end{aligned}
$$

The online version of the original article can be found under doi:10.1007/s11012-012-9686-3.

S. Bennati · P. Fisicaro · P.S. Valvo $(\bowtie)$

Dipartimento di Ingegneria Civile e Industriale,

Università di Pisa, Largo Lucio Lazzarino, 56126 Pisa,

Italy

e-mail:p.valvo@ing.unipi.it 